\title{
Characterizations of dynamic material properties on compact pulsed power generator CQ-4
}

\author{
Guiji Wang ${ }^{1, *}$, Binqiang Luo $^{1}$, Xuping Zhang ${ }^{1}$, Jintao Cai $^{1}$, Tao Chong ${ }^{1}$, Jianheng Zhao ${ }^{1}$, Xuemiao Chen ${ }^{1}$, \\ Fuli $\mathrm{Tan}^{1}$,Chengwei Sun ${ }^{1}$, Cangli $\mathrm{Liu}^{2}$ and Gang $\mathrm{Wu}^{1}$ \\ ${ }^{1}$ Institute of Fluid Physics, China Academy of Engineering Physics, Mianyang City, 621999, Sichuan Province, P. R. China \\ ${ }^{2}$ China Academy of Engineering Physics, Mianyang City, 621999, Sichuan Province, P. R. China
}

\begin{abstract}
Over last two decades, the techniques of magnetically driven quasi-isentropic compression and launching high velocity flyer plates based on pulsed high current generators have being extensively used to do dynamic material experiments under extreme conditions, such as high pressure, high temperature and high strain rate. A compact pulsed power generator CQ-4 was developed to do quasi-isentropic compression experiments of materials at Institute of Fluid Physics of CAEP, which can deliver maximum peak current of about 4 MA to short-circuit loads and produce approximate $100 \mathrm{GPa}$ pressure on the metallic samples. On CQ-4, several types of dynamic material experiments have being conducted for equation of states, phase transitions, constitutive relationships, micro-structure evolutions of matter under quasi-isentropic compression and shock loadings. Meanwhile the dynamic behaviors of solid plastic bonded explosives and their components have also being researched for better understanding the interaction of explosive components under stress waves and the hot spot originations and evolutions mechanism of PBX explosives under dynamic loadings. Several typical applications in dynamic material properties were shown in this paper to exhibit the capabilities of CQ-4.
\end{abstract}

\section{Introduction}

Exploring the dynamic material properties at extreme conditions such as high pressure and high strain rate is very significant in many scientific and engineering fields [1-3], for example, in the target designs of laser inertial confined fusion, the insights into the equation of state and strength of polythene shell and its Rayleigh-Taylor instability growth during laser loading are one of the critical factors affecting the success of target ignition [45]. Another typical example is the shielding of space shuttle and station against high-velocity impacting of debris or micro meteorite at average velocity of 10-15 $\mathrm{km} / \mathrm{s}$ [6-7]. Over last several decades, multi-techniques of dynamic loadings were developed to unravel the characteristics of materials at different loading pressures and strain rates, such as one-stage and two-stage gas gun, laser irradiation, explosive driving high velocity plates and magnetically driven loadings [8-11]. Recent many experiments demonstrate that the technique of magnetically driven ramp wave (quasi-isentropic) compression and launching high velocity flyer plates based on pulsed high current generators is a very precise experimental method to do researches on the dynamic material properties under ramp wave and shock compression [12-15], which can realize loading pressures from tens of GPa to several TPa and strain rates from $10^{4} / \mathrm{s}$ to $10^{7} / \mathrm{s}$. Since 2003, the compact CQ series pulsed power generators for ramp wave and shock experiments were developed to cover loading pressures from tens of GPa to several hundred GPa at Institute of Fluid Physics(IFP), China Academy of Engineering Physics(CAEP) [16,17]. CQ-4 is one of them, which can deliver pulsed currents with peak value of 3 4 MA and rise time of $470 \mathrm{~ns} \sim 600 \mathrm{~ns}$ to short circuit loads. Using optimally shaped electrode panels, ramp wave pressures up to $\sim 100 \mathrm{GPa}$ can be produced in copper and higher impedance metals on CQ-4 [18, 19]. Alternately using the magnetic pressure to launch aluminum flyer plates can produce velocities $>15 \mathrm{kms}-1$ [20]. In the following sections, we will mainly focus on several types of characterizations of dynamic material properties on compact pulsed power generator CQ-4.

\section{Experimental techniques on CQ-4}

Two typical types of loading modes with different thermodynamic paths including ramp wave and shock compressions were developed to do experimental researches on dynamic material properties on CQ-4. The schematics of magnetically driven ramp wave and high velocity flyer plate shock compressions are presented in Figure 1. 


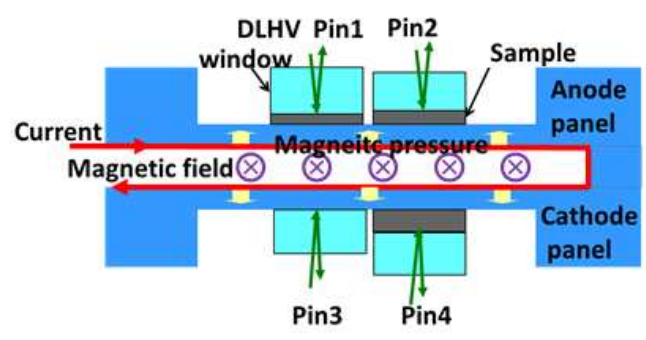

(a)

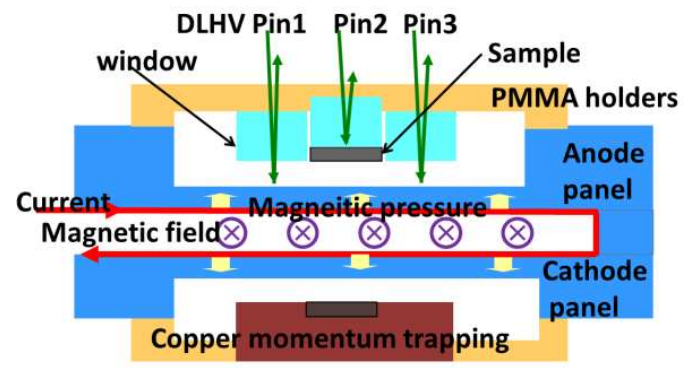

(b)

Fig. 1. The schematic diagrams of magnetically driven experiments on CQ-4. (a) magnetically driven ramp wave experiments, and (b) planar shock compression experiments by magnetically driven high velocity flyer plates.

For magnetically driven ramp wave loading experiments shown in Fig. 1(a), due to the relatively long rise time over which pressure increases, the stress wave structure travelling through a target can be clearly observed through velocimetry measurements. In addition, controlling the rise time of the current pulse alters the loading strain rate, readily enabling experiments in a regime of $10^{4}-10^{6} \mathrm{~s}^{-1}$. The shaped electrode panels ensure that the magnetic field is uniformly distributed across the loading surface, and that pressure propagates as a near planar wave through the thickness of panels $[18,20]$. The dependence of the pressure loading with time can be calculated by measuring the velocity of an electrode panel through an impedance matched window (PIN3 in Figure1) and applying a backwards integration method [21]. This and other velocities are measured by a Dual Laser Heterodyne Velocimetry (DLHV) system with accuracies of $1 \%$ [22]. Measuring the velocity of the rear surface of different thickness targets (PINS 1, 2, and 4) enables the Lagrangian sound speed through the targets to be calculated at different particle velocities. This then enables recovery of the stress-strain or pressure-specific volume relationships, and other dynamic material properties by means of forward calculation methods such as the dynamic strength and phase transition dynamics of materials.

As shown in Fig.1(b), in the shock wave experiments driven by magnetically accelerated flyer plates, DLHV pins are used to record the shock arrival times with time resolution $<1 \mathrm{~ns}$. The initial shock time in target and flyer plate velocity are recorded by pins 1 and 3. Pin 2 record the shock wave transition time and velocity profiles from the back surface of target. Shock wave velocity is determined by the thickness of sample and shock wave transit time. The particle velocity is deduced from the flyer velocity and interface velocity by the method of impedance matching.

\section{Typical applications in dynamic material properties on $C Q-4$}

\subsection{Isentropes and verification of conventional equation of states}

The technique of magnetically driven ramp wave loading has been extensively used to probe the isentropesof materials at off-Hügoniot statesand finally determine their equations of state (EOSs), which are necessary for us to do hydrodynamic simulations. Including the strength data which exist in the materials under ramp wave loadings even up to several hundred $\mathrm{GPa}$, the isentropes can be used to verify the offHügoniot characteristics of conventional EOSs. Based on CQ-4, the isentrope and strength of diverse materials have being measured by means of magnetically driven ramp wave loading technique. Here the example of tantalum (Ta) under ramp wave loadings is taken to illustrate this operation. Figure 2 gives the typical interface velocity profiles between Ta samples and LiF windows. Both backward integration [21] and characteristic inverse integration [23] methods are used to explore the longitudinal stress-volume relation of $\mathrm{Ta}$ under ramp wave loading, and the Steinberg-CochranGuinan (SCG) constitutive model is introduced to obtain the flow stress of Ta under quasi-isentropic compression up to $100 \mathrm{GPa}$. The details of calculations can be seen in our published article [19]. The Longitudinal stress, quasi-isentropic pressure and isentropic pressure versus volume relationships are presented in Figure3, in which the ICE quasi-isentropic pressure is obtained by subtracting the deviator stress from the measured longitudinal stress. Then, the ICE isentropic pressure is deduced from the ICE quasi-isentropic pressure by subtracting the pressure increment induced by the irreversible energy dissipation.

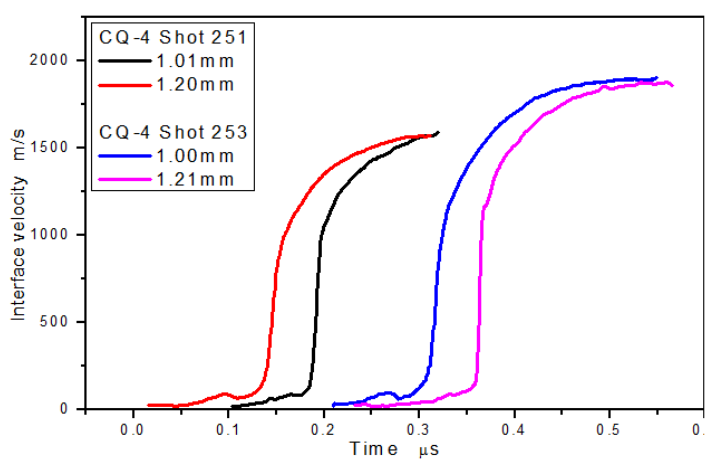

Fig. 2. The measured interface velocity profiles between Ta samples and LiF windows on CQ-4.

Taking the measured isentrope of Ta as the reference line, we verify several conventional theoretical EOSs such as Grüneisen, Vinet, Birch, PUFF and Tillotson 
EOSs.The detailed comparisons are presented in Figure 4.

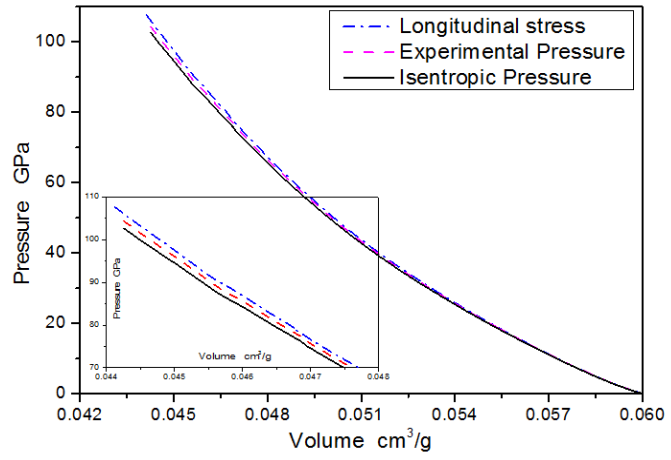

Fig. 3. The Longitudinal stress, quasi-isentropic pressure and isentropic pressure versus volume relationships inferred from the experimental velocity histories.

In Fig.4, Based on the Gruneisen EOS with its parameter Gamma proportional to volume, the theoretical isentrope can be calculated with a linear experimental Hugoniot as a reference, and theoretical Hugoniot curves can also be calculated oppositely with the ICE isentropes discussed above as references. Regardless if the experimental Hugoniot or isentropes are used as references, the Gruneisen EOS always shows good agreement for different compressive states in the pressure-volume plane. Furthermore, the ICE quasiisentrope, even the ICE longitudinal stress curve, can be used as a reference in Gruneisen EOS instead of the ICE isentrope. No marked deviation is induced by this substitution, though this substitution is not rigid in the field of thermodynamics, particularly in the calculation of specific energy. The Vinet EOS has a universal $(\mathrm{p}, \mathrm{v}, \mathrm{T})$ expression for all types of solids under compression without phase transitions, from which a theoretical isentrope has been developed in this work. We find that this isentrope yields very good agreement with the ICE isentrope, but is lower than the ICE longitudinal stress. This phenomenon is reasonable and related to the fact that the Vinet EOS describes the behaviors of materials under hydrostatic compression, particularly isothermal materials, and does not reflect material strength and dissipation effects.

Theoretical isentropes are also deduced from some conventional EOSs, such as the Tillotson, PUFF, and Birch-Munarghan EOSs. We compare these isentropes with the ICE isentropes. A good agreement is achieved in the range of $0-100 \mathrm{GPa}$, but the difference increases gradually at higher pressures. The Birch-Murnaghan isentrope is higher than the Tillotson and PUFF EOSs, and the PUFF isentrope is lower than the ICE isentropes at high pressures. The Tillotson isentrope provides a better relative agreement with the ICE isentropes.

\subsection{Dynamic strength and constitutive relationship}

Strength of material is an important physical parameter which reflects the ability to resist shear deformation. The experimental data show that the strength of material exists under ramp wave loadings even up to several hundred GPa [24]. On CQ-4, two types of techniques for measuring the dynamic strength at high pressures were developed. One is the self-consistent method under ramp wave compression [25], in which the in situ particle velocities of samples with different thicknesses under ramp wave loading and unloading are used to derive the dynamic strength of material at different pressures. The other technique is more direct measurement of material strength under high pressure-magnetically applied pressure-shear (MAPS) [26]. The MAPS technique developed on CQ-4 is shown. Figure 5 presents the schematic of MAPS experiments and Figure 6 gives the typical application in directly measuring the dynamic strength of aluminum.
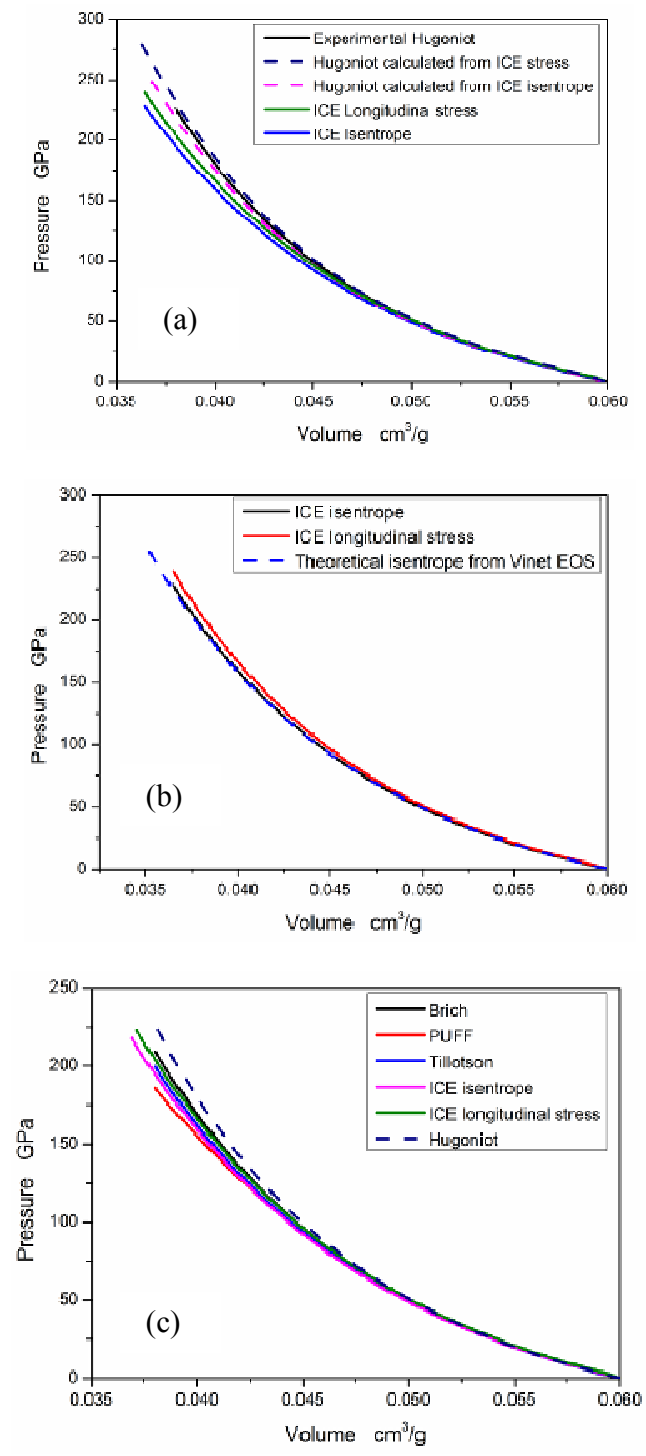

Fig. 4. The comparisons of experimental and conventional EOS calculated isentropes. (a) Verification of global suitability for the Gruneisen EOS using the ICE isentrope or the longitudinal stress as a reference. The Hugoniot data cited from Marsh (1980) [31], (b) Verification of global suitability for the Vinet EOS and (c) Verification of global suitability for other conventional EOSs. 
As show in Figure 6, the measured strength of polished aluminum agrees well with the previous data measured using the self-consistent method. Due to the difference of initial plastic strain, the strength of cold rolled aluminum is bigger than that of polished aluminum. Meanwhile, the strength of polished and cold rolled aluminum increases with the loading pressures.

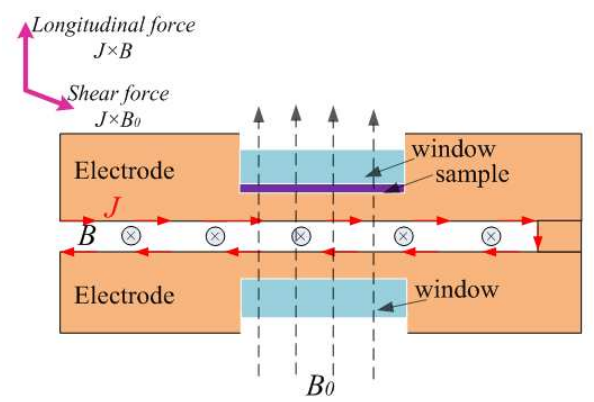

(a)

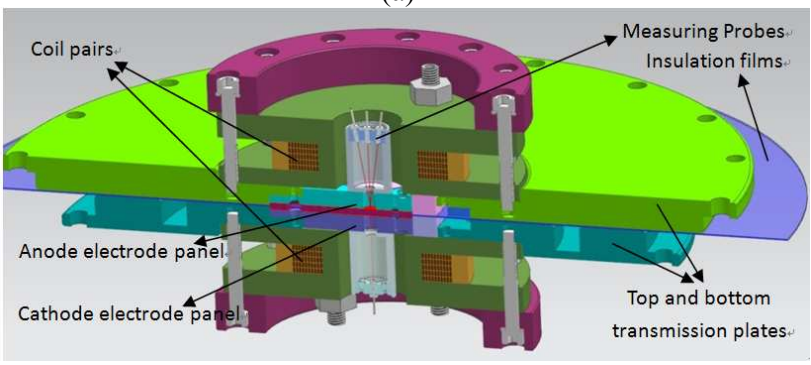

(b)

Fig. 5. Schematic of magnetically applied pressure shear loading(a) and 3D drawing of loading regime for MAPS experiments (b).

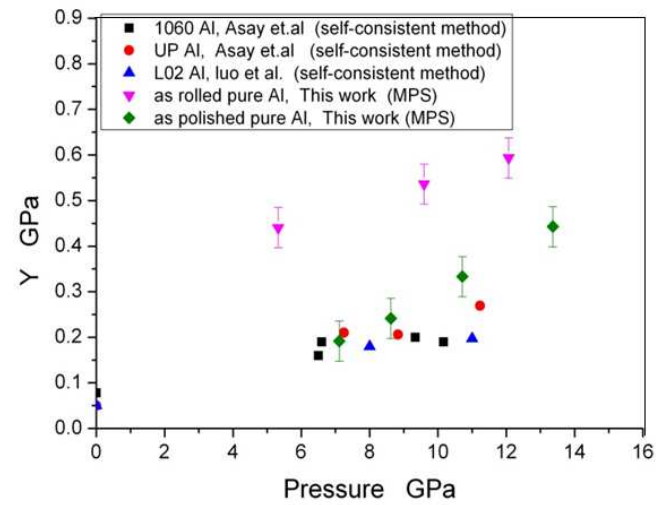

Fig. 6. The dynamic strength of Rolled (purple) and polished (green) pure aluminum directly measured by MAPS on CQ-4.

\subsection{Phase transition and its dynamics}

Due to its process loading with long rise time under ramp wave compression, it is more superior to probe some tiny or abrupt changes in the wave profiles. Therefore, the technique of magnetically driven ramp wave loading is a very useful tool to research the polymorphic phase transition of materials and related dynamics. On CQ-4, diverse materials such as bismuth, iron, zirconium and tin were taken to do experiments for obtaining some insights into their phase transition and dynamics including multi-phase EOS, strength model and non-equilibrium dynamic equation of phase transition [26, 27]. Figure 7 gives two typical experimental and calculated data.
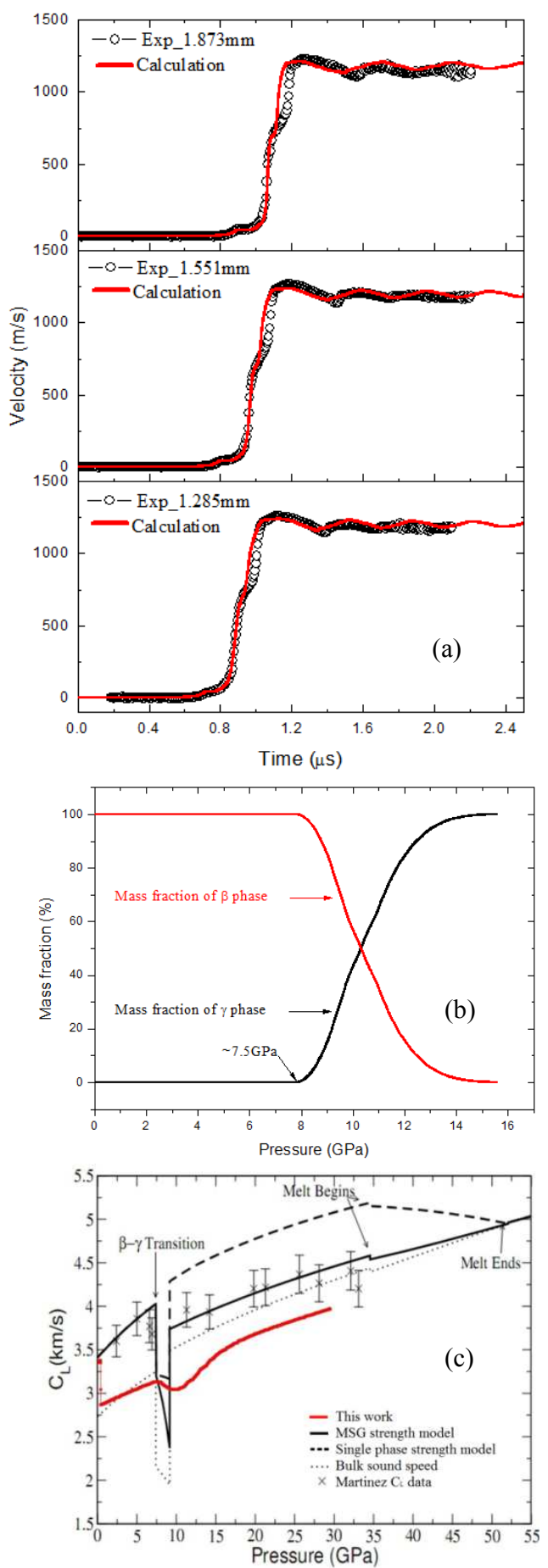

Fig. 7. The typical phase transition experiment under ramp wave loadings on CQ-4. (a)the comparisons between experimental and calculated interface particle velocities between Tin samples and LiF windows, (b) the evolution of different phase and (c) comparisons of Lagrangian sound speed of this simulation with other researchers' experimental and calculated results $[29,30]$.

\subsection{Dynamics of un-reacted solid explosives}

One of the notablecharacteristics for ramp wave loadings is that the temperature rise is very low in the samples. So this technique provides a good tool to 
research the dynamic behaviors of solid explosives in a very wide pressure range from $1 \mathrm{GPa}$ to tens of $\mathrm{GPa}$ [30], which is impossible for shock compression to do. Meanwhile, we can also research the reaction rate of solid explosives by putting six or more explosive samples with different thicknesses in one ramp wave experiment shot. In this experiment, the things of explosive reaction from zero reaction to part reaction and then to final complete reaction can be observed by wave profiles. The loading pressure of near $20 \mathrm{GPa}$ is produced in the un-reacted solid explosives on CQ-4. Figure 8 shows our typically experimental results.

By means of Lagrangian analysis, impedance matching and backward integration methods, the isentrope, strength model and reaction rate of solid explosive can be obtained, which are necessary to do the hydrodynamic simulations of explosive detonation or ammunition safety.

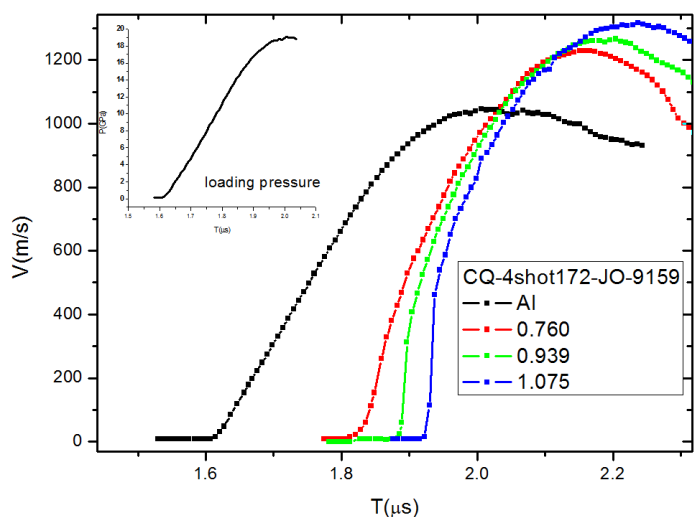

(a)

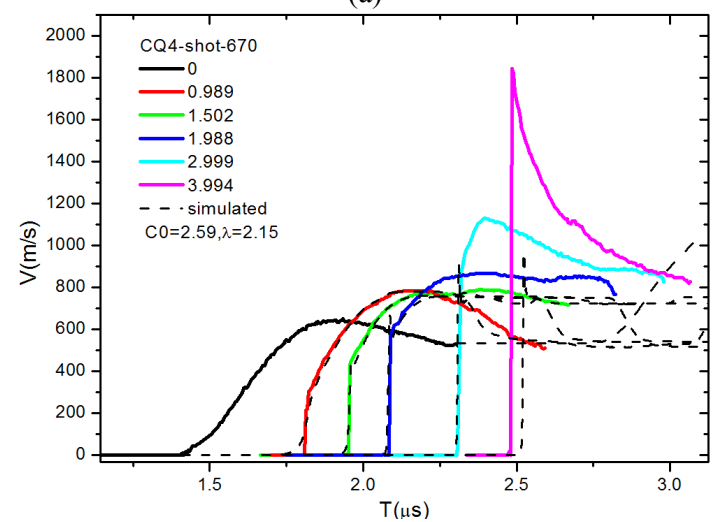

(b)

Fig. 8. Typical experimental data of some solid explosives under ramp wave loadings on CQ-4. (a) interface particle velocities between samples and $\mathrm{LiF}$ windows for different sample thicknesses, and (b) the reaction extent of solid explosive samples with different thicknesses.

\section{Summary}

There are many other applications on CQ-4. For example, many shock compression experiments by magnetically driven high velocity flyer plates had been done to reveal some natures of materials such as the EOS and strength of NiTi alloy [31], Refractive index and molecular polarizability of polystyrene and multi-shock properties of acetone.
In last ten years, the technique of magnetically driven ramp wave loading was developed for dynamic material properties on the compact pulsed power generator CQ-4 at IFP of CAEP. The aforementioned applications on CQ-4 have being conducted to understand the dynamic behaviors of materials at macroscale in experiments and simulations. In the following, we will do many researches in meso and micro-scale simulations besides some detailed metallographes of recovered samples from CQ-4 experiments. Of course, if possible, we hope that we can integrate our compact loading generators into advanced X-ray light sources such as synchrotron or free electron laser for in situ detections of materials under mesoscale or microscale.

The authors wish to thank Mr. Chao Xu,RongjieShui, Xiao Ma, and Shunyi Deng at IFP for their dedications to experimental operations on CQ-4. This work was supported by the National Natural Science Foundation of China (Contract No. 11327803, 11502252), the project of Youth Innovation of Science and Technology of Sichuan Province (Contract No. 2016TD0022).

\section{References}

1. R. F. Smith, J. H. Eggert, R. Jeanloz, T. S. Duffy, D. G. Braun, J. R. Patterson, R. E. Rudd, J. Biener, A. E. Lazicki, A. V. Hamza,J. Wang, T. Braun, L. X. Benedict, P. M. Celliers\& G. W. Collins, Nature, Vol.511, 17 (2014)

2. M. Millot, N. Dubrovinskaia, A. Černok, S. Blaha, L. Dubrovinsky, D. G. Braun,P. M. Celliers, G. W. Collins, J. H. Eggert, R. Jeanloz, Science, Vol.347, 6220(2015)

3. M. D. Knudson, M. P. Desjarlais, A. Becker, R. W. Lemke, K. R. Cochrane,M. E. Savage, D. E. Bliss, T. R. Mattsson, R. Redmer, Science, Vol.348, 6242 (2015)

4. Hye-Sook Park, B. A. Remington, R. C. Becker, J. V. Bernier, R. M. Cavallo,K. T. Lorenz, S. M. Pollaine, S. T. Prisbrey, R. E. Rudd, and N. R. Barton, PHYSICS OF PLASMAS 17, 056314 (2010)

5. Bruce A. Remington, R. Paul Drake,Dmitri D. Ryutov, Reviews of Modern Physics, vol. 78, 3 (2006)

6. K. Thoma, F. Schafer , S. Hiermaier, E. Schneider, Advances in Space Research 34 (2004)

7. E. L. Christiansen, J. H. Kerr, H. M. De La Fuente, W. C. Schneider, International Journal of Impact Engineering, 23 (1999)

8. A. C. Charters, International Journal of Impact Engineering, 5 (1986)

9. S. P. Marsh and T. H. Tan. in: Shock Waves in Condensed Matter-1991[C], eds. S. C. Schmidt, J. Forbes and R. Dick, Elsevier Science Publishers, BV (1992)

10. M.D.Knudson, R.W. Lemke et al., Journal of Applied Physics 94, 7 (2003)

11. J. Edwards, K. T. Lorenz, B. A. Remington et al., Physical Review Letters, Vol.92, 7(2004)

12. M. D. Knudson and M. P. Desjarlais, PHYSICAL REVIEW B 91, 224105 (2015) 
13. R. Cochrane, R. W. Lemke, Z. Riford, and J. H. Carpenter, Journal of Applied Physics 119, 105902 (2016)

14. Binqiang Luo, Mu Li, Guiji Wang, Fuli Tan, Jianheng Zhao, Chengwei Sun, Mechanics of Materials 114 (2017)

15. Guiji Wang, Jianheng Zhao, Hongping Zhang, Chengwei Sun, Fuli Tan, Ganghua Wang, Jianjun Mo, Jintao Cai, and Gang. Wu, Eur. Phys. J. Special Topics 206(2012)

16. Guiji Wang, Chengwei Sun, Fuli Tan, Jianheng Zhao, Ning Zhang, Cangli Liu,Jianjun Mo, Ganghua Wang, and Xiaosong Wang, REVIEW OF SCIENTIFIC INSTRUMENTS 79, 053904(2008)

17. Guiji Wang, Xuemiao Chen, Jintao Cai, Xuping Zhang, Tao Chong, Binqiang Luo, Jianheng Zhao, ChengweiSun, Fuli Tan, Cangli Liu and Gang Wu, Review of Scientific Instruments 87, 065110 (2016)

18. Guiji Wang, Binqiang Luo, Xuping Zhang, Jianheng Zhao, Chengwei Sun, FuliTan,Tao Chong, Jianjun Mo, Gang $\mathrm{Wu}$, and Yanhui Tao, REVIEW OF SCIENTIFIC INSTRUMENTS84, 015117 (2013)

19. Binqiang Luo, Guiji Wang, Jianjun Mo, Hongping Zhang, Fuli Tan, Jianheng Zhao, Cangli Liu, and Chengwei Sun, Journal of Applied Physics 116, 193506 (2014)

20. Xuping Zhang, Guiji Wang, Jianheng Zhao, Fuli Tan, Binqiang Luo and Chengwei Sun, Review of Scientific Instruments 85, 055110 (2014)

21. Hongping Zhang, Chengwei Sun, Mu Li and Jianheng Zhao, Chinese Journal of theoretical and applied mechanics, vol.43,1( 2011)
22. T. J. Tao, J.D. Weng, X. Wang, Opto-Electronic Engineering, Vol.38,10(2011)

23. J.-P. Davis, Report SAND2007-4984, 2007

24. J. L. Brown, C. S. Alexander, J. R. Asay, T. J. Vogler, D. H. Dolanand J. L. Belof, Journal of Applied Physics 115, 043530 (2014)

25. J. L. Brown,M. D. Knudson,C. S. Alexander,J. R. Asay, Journal of Applied Physics116, 033502(2014)

26. B.Q. Luo, X.M. Chen, G.J. Wang, F.L. Tan, G.H. Chen, J.H. Zhao, C.W. Sun, International Journal of Impact Engineering 100 (2017)

27. T. Chong, G.J. Wang, F. L. Tan et al., Sci Sin-Phys. Mech. Astron, Vol. 44, 4 (2014, in Chinese)

28. T. Chong, Z.P. Tang, F.L. Tan, G.J. Wang, J.H. Zhao, Chines Journal of High Pressure Physics, Vol.32,1 (2018)

29. G. A. Cox, Shock Compression of Condensed Matter2005, edited by M. D. Furnish, M. Elert, T.P. Russell et al., American Institute of Physics, 208(2006)

30. E. Martinez and J. M. Servas, Shock Compression of CondensedMatter-2001, edited by M. D. Furnish et al., AIP Conf. Proc. 620 (2002)

31. G.J. Wang, J.T. Cai, H.P. Zhang, F. Zhao, F.L. Tan, and G. Wu, Eur. Phys. J. Appl. Phys., 60: 21001 (2012)

32. Xuping Zhang, Guiji Wang, Binqiang Luo, Simon N. Bland, Fuli Tan, Feng Zhao,Jianheng Zhao, Chengwei Sun, Cangli Liu, Journal of Alloys and Compounds, 731 (2018) 